\title{
Quo vadis, Gesundheitswesen?
}

\author{
Kein Gesundheitssystem kann auf Dauer unbegrenzte Leistungen \\ mit begrenzten Finanzmitteln erbringen. Reformen sind unab- \\ dingbar. Aber beim derzeitigen Reformstau sind Einschränkungen \\ sowohl für die Patienten, aber auch für die Ärzte unausweichlich.
}

\begin{abstract}
m Laufe der letzten Jahre hat sich die kassenärztliche Vergütung der niedergelassenen Ärzte um 20-30\% verringert; bei einzelnen Gruppen mehr, bei anderen weniger. Die Gründe sind vielfältig: Budgetierung, Kopfpauschalen, Kostenstrukturausgleich, außerbudgetäre Honorierung aufgrund gesetzlicher und vertraglicher Regelungen. Diese Faktoren bedingen Personalentlassungen und Ausdünnung des Leistungsangebots, wobei zeitintensive Leistungen wie etwa Arzneimitteltestungen mit Individualanfertigungen oder aufwendige Provokationstests nicht mehr angeboten werden können.
\end{abstract}

\section{Unfreiwilliges Teilzeitmodell}

„Budgetferien“ 14 Tage vor Quartalsende sind bei vielen Kollegen fast eine Selbstverständlichkeit, denn wer möchte gerne bei Budget-Ausschöpfung - und ohnehin geschmälertem Honorar - die dann noch entstehenden Kosten selbst bezahlen. Die Folge ist, dass Terminvergaben zu Untersuchungen erst in drei Monaten „üblich“ sind und zu heftigen Auseinandersetzungen des Empfangspersonals mit den Patienten führen.

Weitere Folterinstrumente der KVen wie Plausibilitäts- und Wirtschaftlichkeitsprüfungen erhöhen den Frust. Die Widersprüche nehmen zu, die Auseinandersetzungen mit der $\mathrm{KV}$ verschlingen immer mehr Zeit. Der Niedergelassene muss sofort antworten, alles belegen und beweisen, sonst drohen Strafen mit Honorarabzug. Die KV nimmt sich zur Erledigung entsprechender Fälle gerne ein bis zwei Jahre Zeit. Eine Entschuldigung bei zugegebenen Fehlern der KVen gibt es kaum.

Mit schöner Regelmäßigkeit wird die Einführung der "Wunderwaffe“ EBM 2000 Plus verschoben. Derzeit wird das Jahr 2003 für die Einführung gehandelt. Ganz abgesehen davon stellt sich die Frage, ob „Regelleistungsvolu- mina" etwas wesentlich anderes darstellen als eine Budgetierung.

Das Wort IGEL-Leistung und deren Umsetzung im Praxis-Alltag mit immer größer werdenden Katalogen leuchtet ein. Aber welcher Patient auf dem Land, in einer Kleinstadt oder kleinen Großstadt kann für die IGEL-Leistungen und für die dort enthaltenen allergologischen Fragestellungen gewonnen werden? In Großstädten ist es vielleicht einfacher.

Ein anderer Punkt ist der „Medikamententourismus". Die Patienten nehmen z.T. 80 km Fahrt in Kauf, um ein Dosieraerosol zu bekommen, weil „der Hausarzt es nicht mehr verschreibt". Die Allergologen tragen schon die hohen Kosten für die Hyposensibilisierungslösungen und tun gut daran, diese Besonderheit den KVen zu melden.

\section{Drei-Klassen-Medizin schon längst Realität}

Längst besteht in der medizinischen Versorgung eine Drei-Klassen-Medizin: Der Privatpatient bekommt eine Medikation nach dem Stand der derzeitigen Wissenschaft. Vielleicht erhalten sogar einige Kassenpatienten diesen Standard, wenn auch mit einigen Einschränkungen. Die übrigen Patienten werden „aus dem Schrank" versorgt und bekommen, wie man immer wieder erleben kann, das, was gerade als Muster vorrätig ist. Trotz Beschwörung aller Rationalisierungsmaßnahmen und unter Ausnutzung aller Synergie-Effekte steht am Ende die Rationierung.

Gegen mehr Transparenz ist nichts einzuwenden. Wie gut wäre es, wenn manch ein Patient wüsste, dass er mit rezeptierten Medikamenten für $500 \mathrm{DM}$ und mehr in der Hand die Praxis verlässt. Die gesamte Entwicklung bedingt, dass Aufklärung und Erklärung der jetzigen Situation häufig das Arzt-Patienten-Verhältnis belasten, wobei viele Pati- enten das Ganze nicht mehr verstehen. Die Politik ist zu feige, um die Realitäten anzuerkennen und suggeriert noch immer die heile Welt. So auch der Tenor vieler Krankenkassen: Wir bezahlen alles, wenn es der Arzt verschreibt!

\section{Alle für einen oder einer für alle?}

Gefeiert wird der Verzicht auf die kollektive Haftung der Ärzte bei Überschreiten des Arzneimittelbudgets. Dafür soll der einzelne Arzt am Ende die wirtschaftliche Dimension seines Verordnungsverhaltens übernehmen, d.h. die Bedrohung bleibt. Sie wird lediglich auf diejenigen verschoben, die viel verordnen „müssen“, weil sie viele und oft chronisch kranke Patienten betreuen. Es sei an dieser Stelle die Frage gestattet: "Welcher Arzt verordnet Medikamente nur so zum Spaß?" Der oben geschilderte "Medikamententourismus" wird also weitergehen.

Die Zukunft wird weiterhin in der Qualität der Arbeit des Einzelnen liegen. Die Fortschritte in der Medizin und insbesondere auch bei der Diagnostik und Therapie allergologischer Erkrankungen können durch keine Regierung, keine Krankenkassen und keine KV aufgehalten werden.

Wie der einzelne Patient in Zukunft auch die neueren medizinischen Leistungen nutzt, wird er kurz über lang weitgehend selbst bestimmen. $\mathrm{Ob}$ dies in Form von Basisversorgung mit privater Zusatzversicherung oder in Form anderer Modelle geschieht, bleibt dahingestellt. Bei Urlaubsreisen und beim Autofahren z.B. werden solche Überlegungen nicht angestellt.

Auch in Zukunft wird bei entsprechend qualifizierter Arbeit die Möglichkeit bestehen, dass im Wettbewerb eine adäquate Honorierung durchsetzbar ist.

Dr. W. Kersten, Moers

Impressum „Der ÄDA informiert"

Verantwortlich für den Inhalt: Dr. Werner Kersten

Redaktion: Dr. Corinna K. Leitnaker

Urban \& Vogel Medien und Medizin Verlagsgesellschaft $\mathrm{mbH} \&$ Co. KG, Neumarkter Straße 43 81637 München 